\title{
AN INVENTORY OF \\ Basic Water Resources Data ISLAND OF HAWAII
}

Report R34

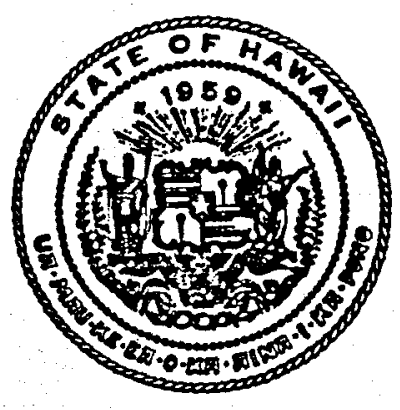

STATE OF HAWAII

Department of Land and Natural Resources Division of Water and Land Development

HONOLULU, HAWAII

February 1970 


\section{DISCLAIMER}

This report was prepared as an account of work sponsored by an agency of the United States Government. Neither the United States Government nor any agency Thereof, nor any of their employees, makes any warranty, express or implied, or assumes any legal liability or responsibility for the accuracy, completeness, or usefulness of any information, apparatus, product, or process disclosed, or represents that its use would not infringe privately owned rights. Reference herein to any specific commercial product, process, or service by trade name, trademark, manufacturer, or otherwise does not necessarily constitute or imply its endorsement, recommendation, or favoring by the United States Government or any agency thereof. The views and opinions of authors expressed herein do not necessarily state or reflect those of the United States Government or any agency thereof. 


\section{DISCLAIMER}

Portions of this document may be illegible in electronic image products. Images are produced from the best available original document. 


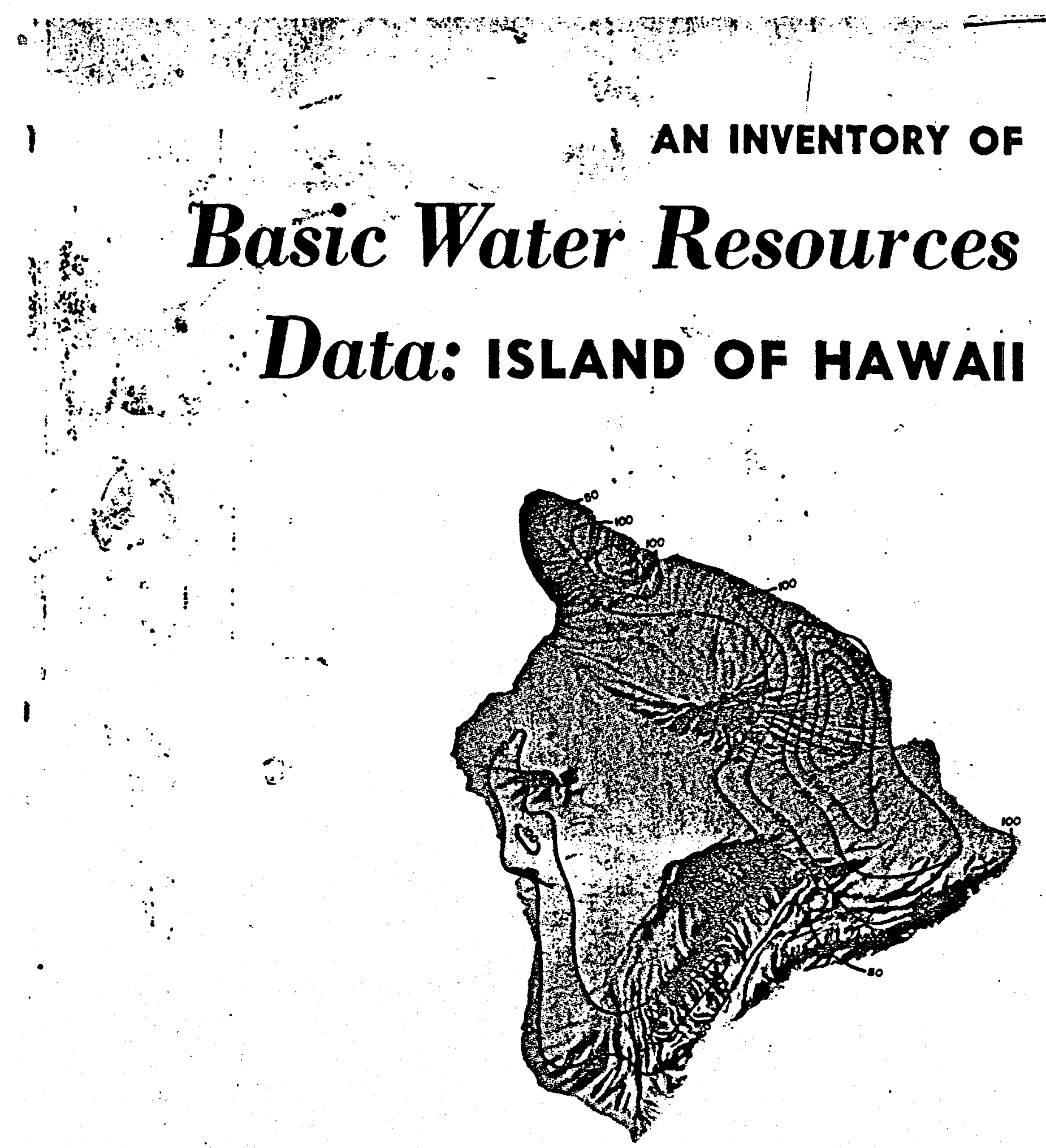

STATE OF HAWAII

Department of Land and Natural Resources Division of Water and Land Development 
- Jield brye quantties of vater of the larger ones are those to ary in Bilo, which Alscharges i callons datly and at Punalim in ch decharges about 25 million

itw long-lerm epring recards, sources ure usually cealed with ittle attention pald to measuring cted Spring Flow Records, pege ischarge from torings used for supplies. Yoathly and snnual

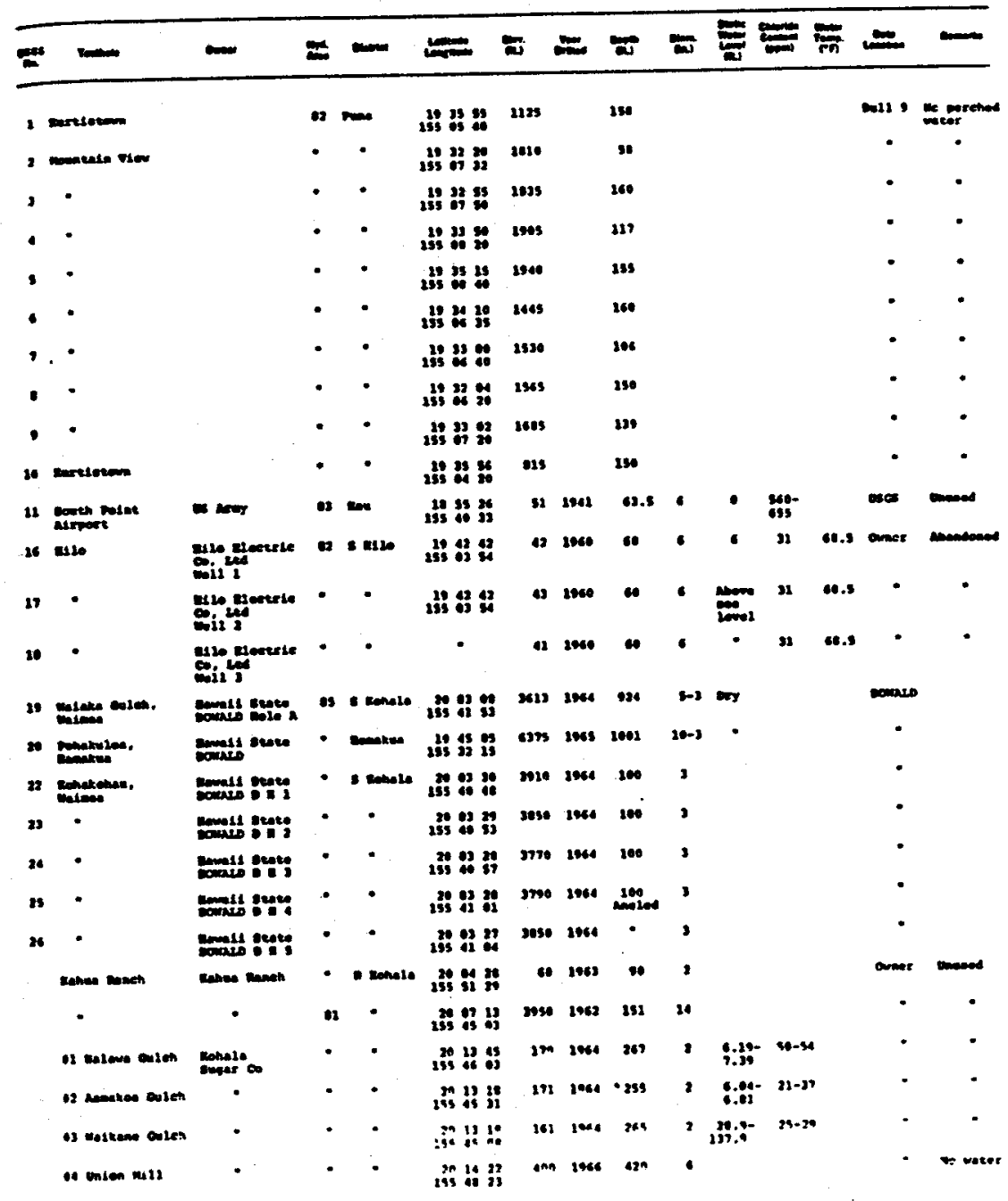

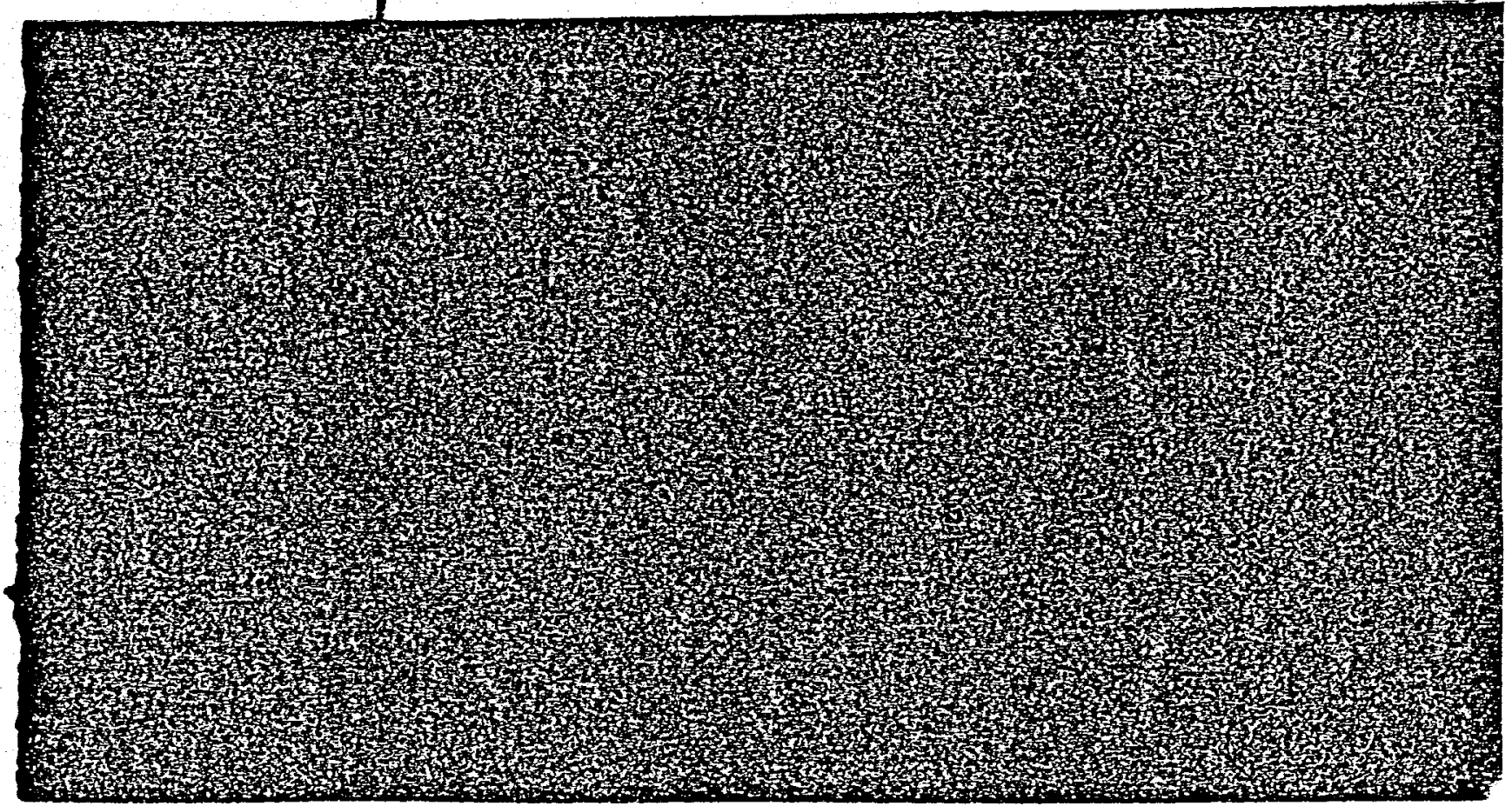




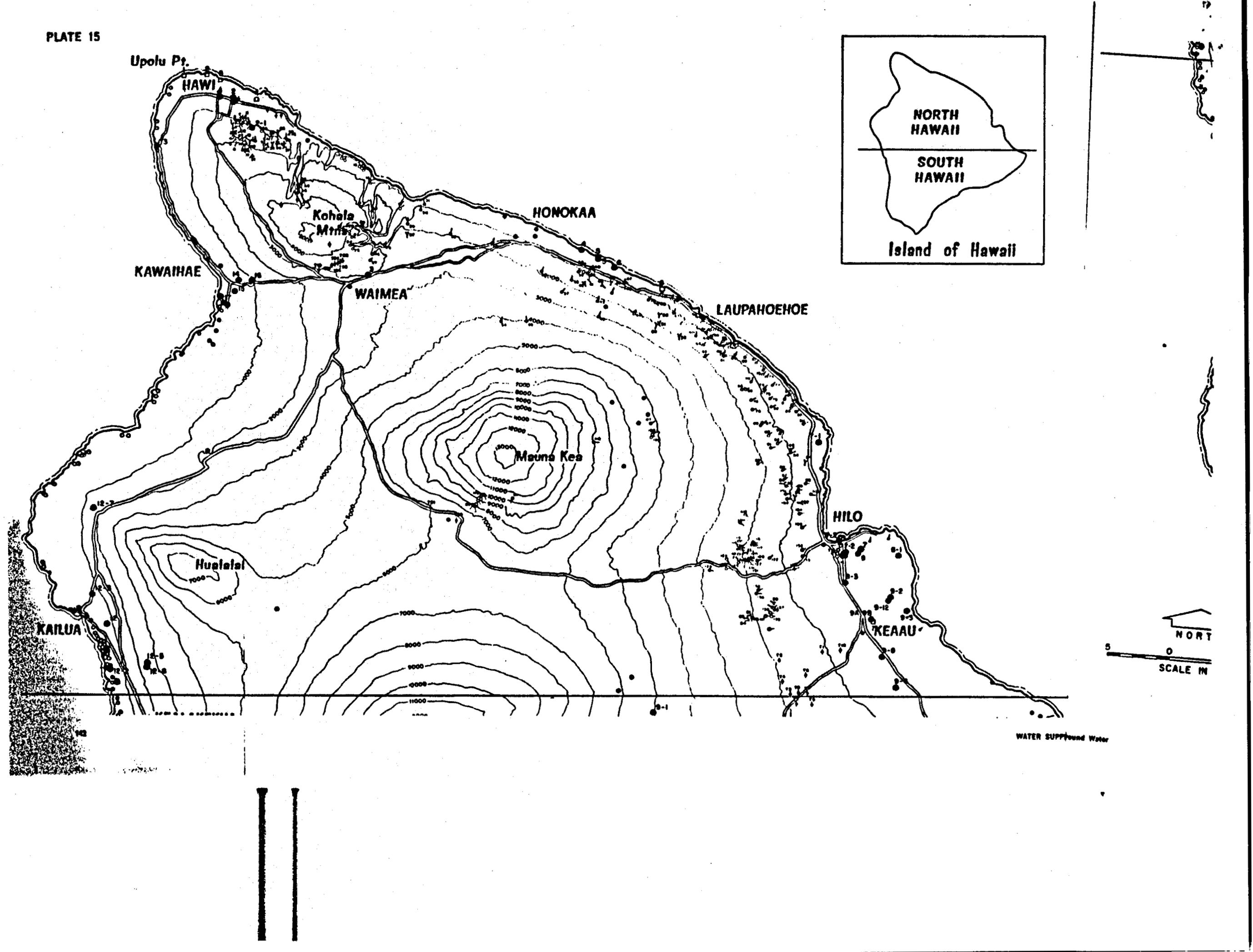




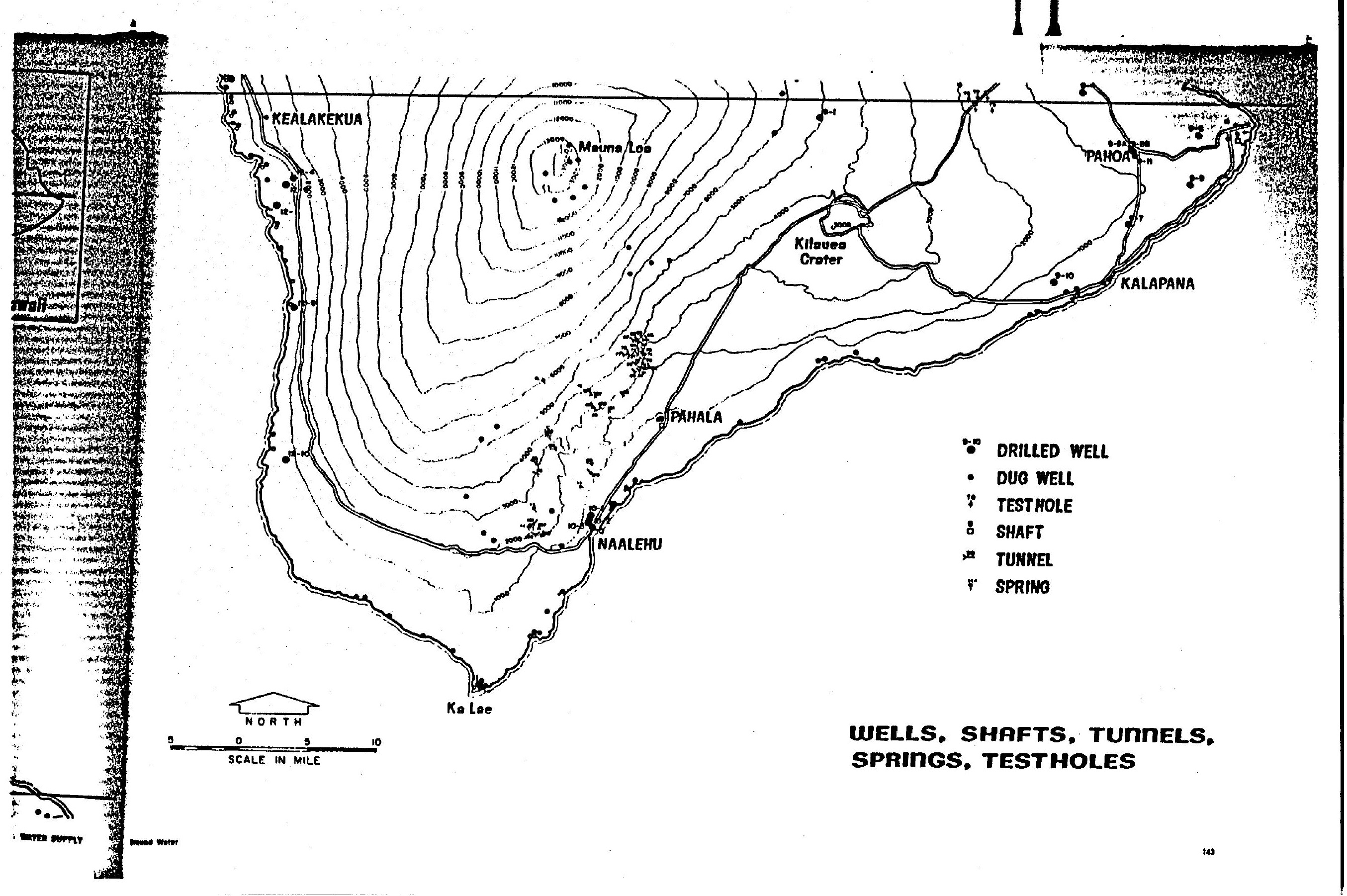



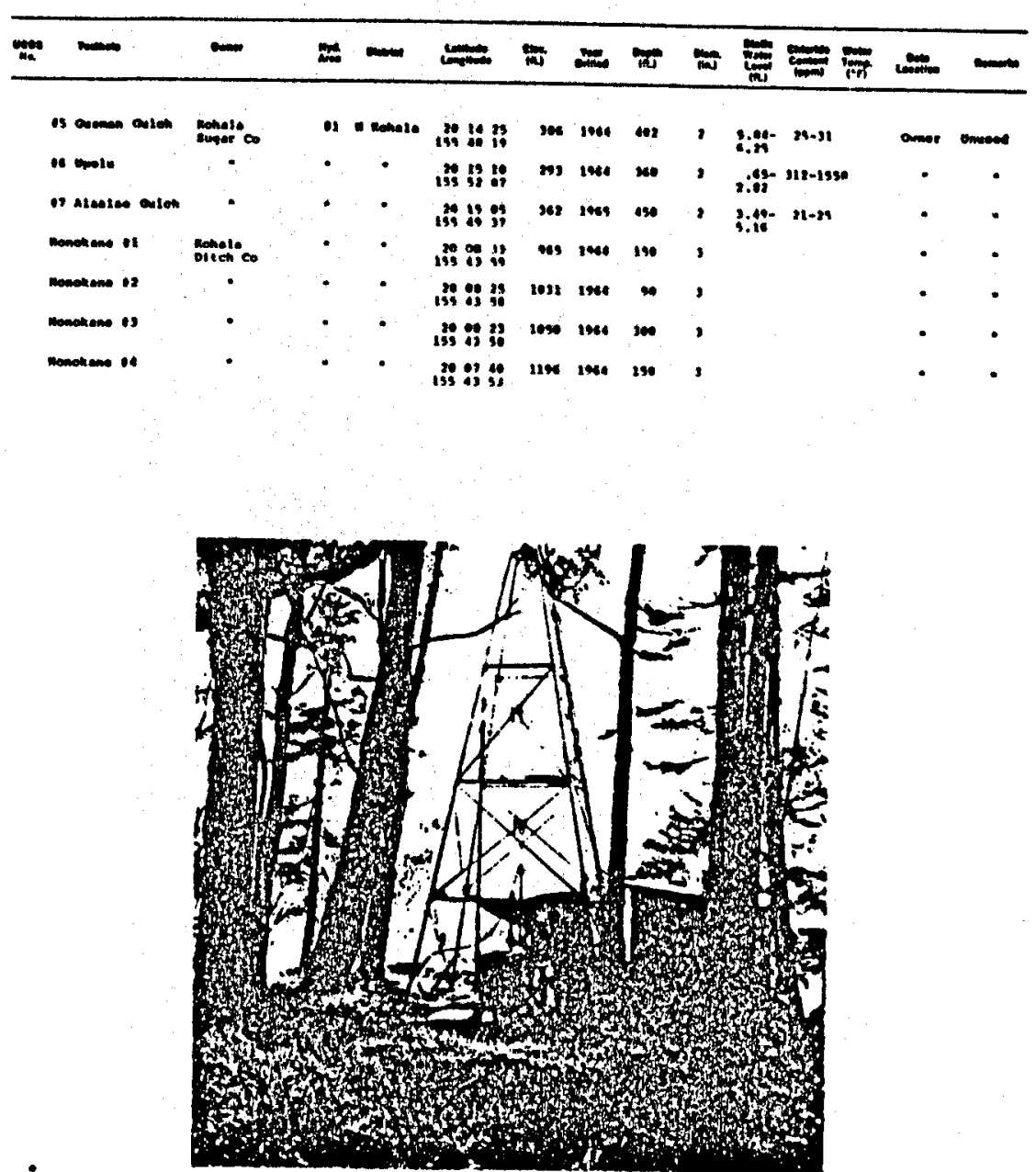

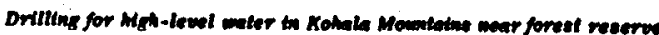

Drilled W'ell Index

\begin{tabular}{|c|c|c|c|c|c|c|c|c|c|c|c|c|c|c|c|}
\hline n & $m$ & - & 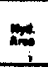 & m & 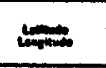 & tit & مי & 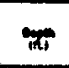 & tin & 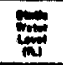 & $=$ & 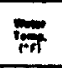 & $m$ & $=$ & $m$ \\
\hline & nom & coloseles" & $n$ & - Eochere & $25314: 48$ & 200 & 1009 & 200 & & ons & & & IIte- & $-\infty$ & \\
\hline & mitin & • & $\cdot$ & $\cdot$ & 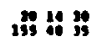 & $\infty$ & 2009 & as & - & & & & Alome & - & exthono- \\
\hline $2-1$ & Matente & • & • & - & 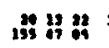 & 303 & 1901 & ses & 13 & 7.1 & 26 & & 110- & • & \\
\hline & $\operatorname{mim}$ & $=$ & • & - Monase & it & 2038 & 2014 & $m$ & - & ort & & & مomon & • & 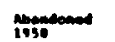 \\
\hline & reowles: & nitin: & $\cdot$ & משח & 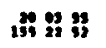 & 199 & 2004 & & $"$ & & & & 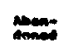 & $\cdot$ & Pitsolen ox \\
\hline & inti1 & $\cdot$ & $\bullet$ & $\bullet$ & $298 \%$ & 319.0 & 2004 & "11..1 & 18 & 1.1 & 20 & & $\cdot$ & $\cdot$ & antentritio \\
\hline & menstem & • & $\cdot$ & $\cdot$ & 13: :28 & MA., & & we., & 12 & ..1 & 213 & & • & - & • \\
\hline & Altione & mit & $"$ & onsto & $138 \%$ & $"$ & 1904 & " & 14 & $\cdot$ & 201 & & iie- & $\cdot$ & ex em mor \\
\hline 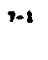 & I: & (1) & $\bullet$ & • & A3 & wo & 1006 & 320 & 13 & & & & isons & $\cdot$ & 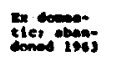 \\
\hline 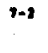 & mitases & 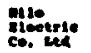 & $\bullet$ & • & 19834 & 96.06 & InI & 201 & 21 & ' & 13 & " & Be- & nomen & \\
\hline & Alowe & il & $\cdot$ & $\cdot$ & 138283 & n & 1004 & N & 16 & , & 100 & & - & noe & \\
\hline & indien: & miscosis & $\because$ & • & $13: 484$ & 30.80 & 1001 & 9 & 10 & .31 & on & & הod & - & \\
\hline & atso & 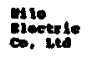 & $\cdot$ & • & 1939848 & ' & 1901 & 201 & 10 & ? & 31 & 81.9 & entioi- & ere & \\
\hline & nomes: & mil & $\cdot$ & $\cdot$ & 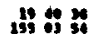 & 201.1 & (26) & 300.1 & $"$ & 39.12 & - & “ & ine- & comenes & \\
\hline & 힘111 & & • & mom & i⿱⺈ & $2 m$ & $2 n$ & 1950 & 12 & 19 & - & 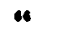 & $\cdot$ & $m$ & \\
\hline & inimi & $\dot{0}$ & : & $\therefore$ & 政 & "I" & m & 950 & 12 & $"$ & ' & “ & $\dot{0}$ & • & \\
\hline & inteon & in it & 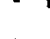 & 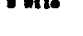 & If 14 & 3000 & 1019 & $\infty$ & " & & & & some & & 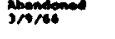 \\
\hline & 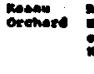 & 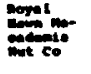 & $\cdot$ & men & If? & $"$ & 2019 & '”' & • & 0.9 & " & $"$ & ide & nom & \\
\hline & Neasen & $=2$ & 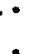 & $\cdot$ & IA :3 :3 & $"$ & 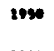 & $\omega$ & 20 & ' & 31 & & - & anor & ex comoetie \\
\hline & 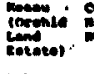 & 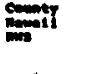 & • & • & 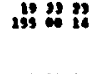 & 98.01 & 2000 & $" n$ & 12 & " & - & $"$ & & comeses & pie mol1 \\
\hline $1 \cdot 1$ & inti1 2-a & • & 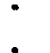 & $\cdot$ & a & mo.t & 1000 & ma., & 2 & 11.0 & ' & $"$ & & $\cdot$ & $72^{20+\infty}$ \\
\hline & nill 2-1" & - & . & - & 19; & 200.9 & 1000 & 3213 & 12 & 1.18 & me & "3 & & - & \\
\hline & 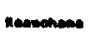 & • & $\cdot$ & • & is 48 : & m1.ns & & nn1.• & $" 1$ & 2.94 & 12 & $"$ & & & 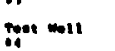 \\
\hline & matpis: & $\cdot$ & • & • & 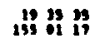 & Mo..06 & & 301.41 & $" 1$ & 16.10 & • & $"$ & & • & 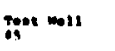 \\
\hline & & & & & & & & & & & & & & 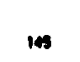 & \\
\hline
\end{tabular}




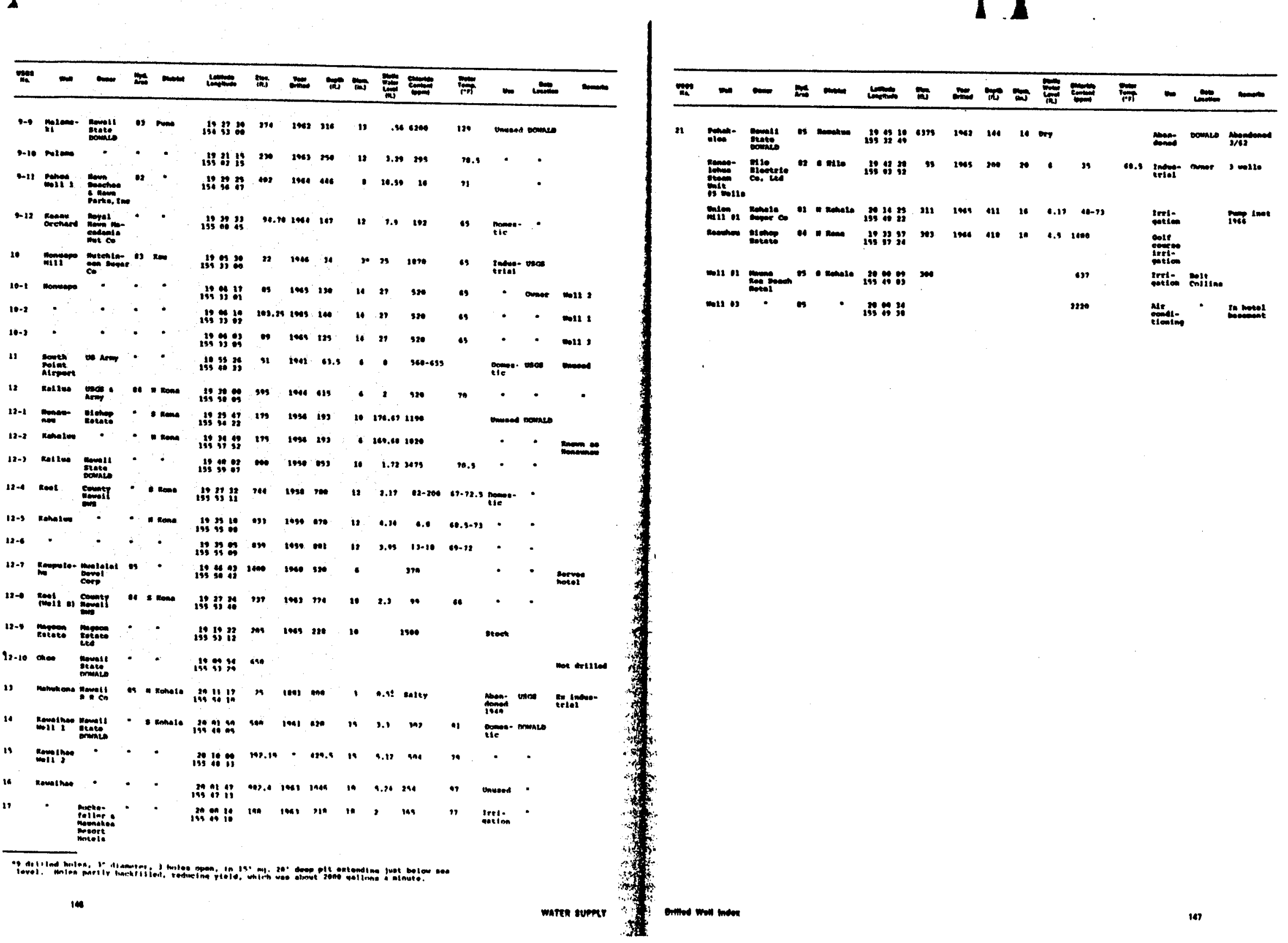


$\|$

Selerted Hell Pumpuge Reconds

$-1=-2=-1=$

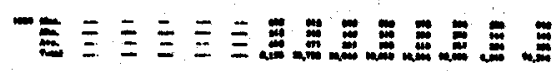

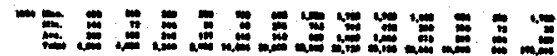

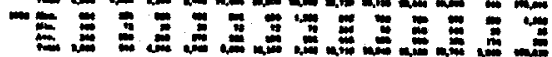
-

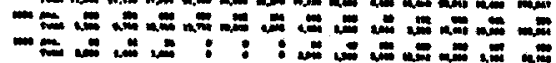

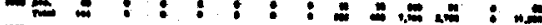

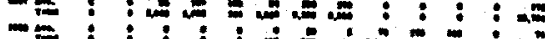

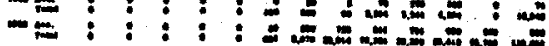

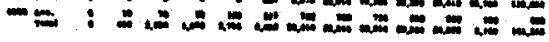

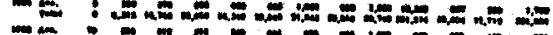

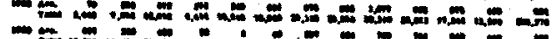

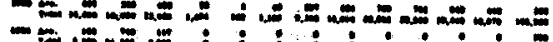
m -

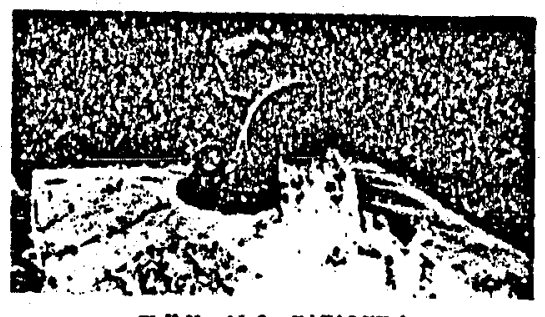

moll No. 12-3 $\rightarrow$ KAmALUD A
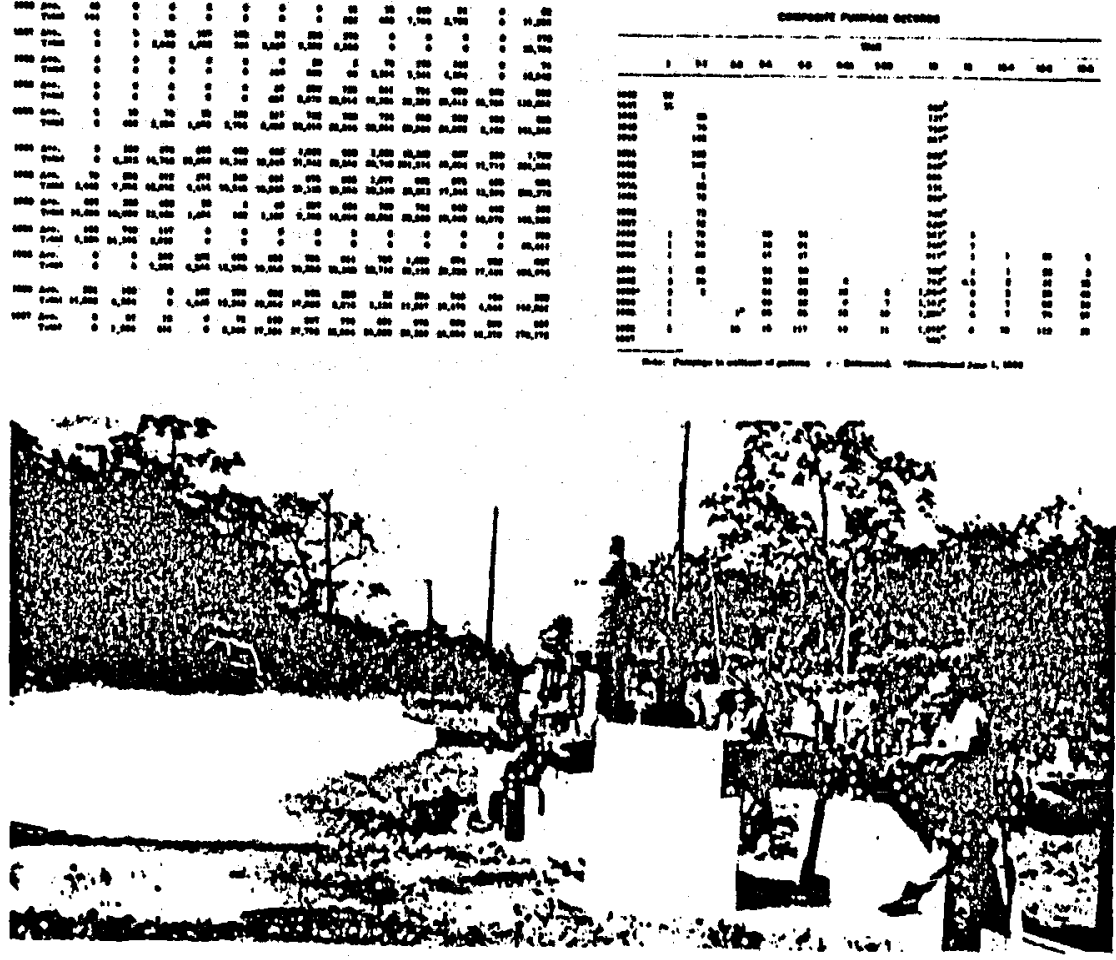

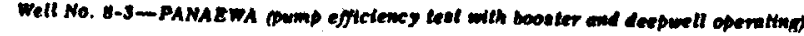

Seleeted Will Pumping Teet Rerowds

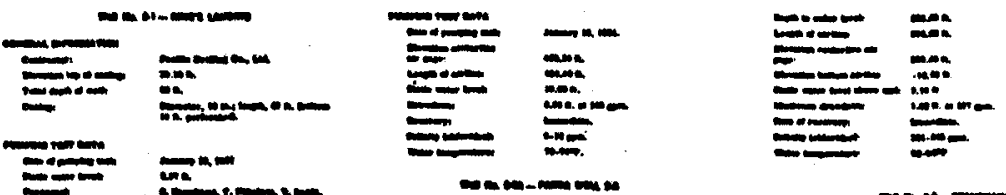

$\equiv=$

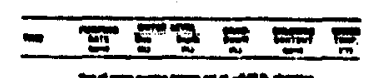

$=\because-\infty \equiv \equiv$

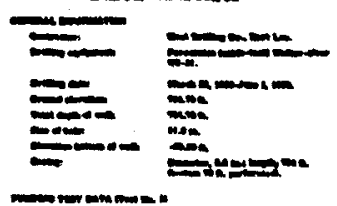

$=-\infty$ an an a

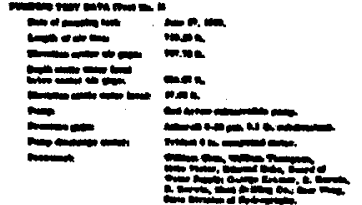

nition $\div=$

$m-\infty-\infty-1=$

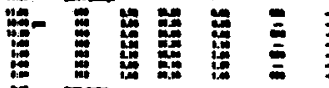

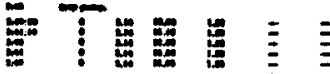

$\pm=5=5=$

in

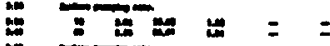

$=\div \div$ : $=$

$\therefore \div-5=$

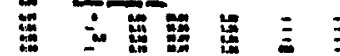

$\div \div$ i

$\div$ - $=5=$

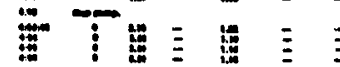
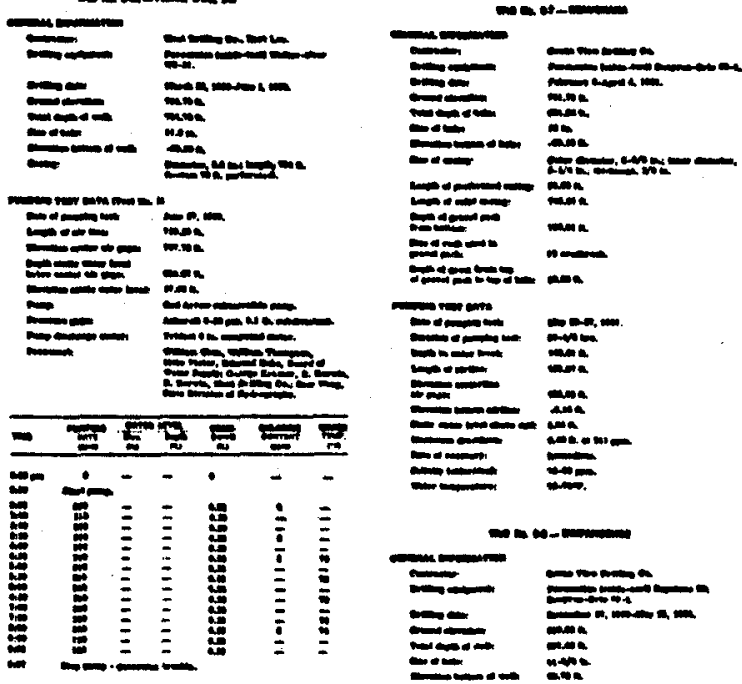

$=-\infty$
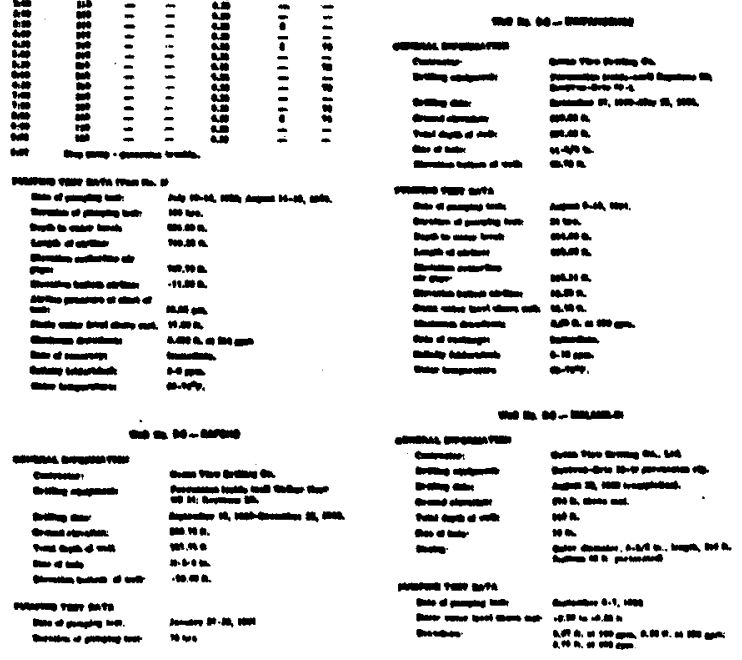

m 


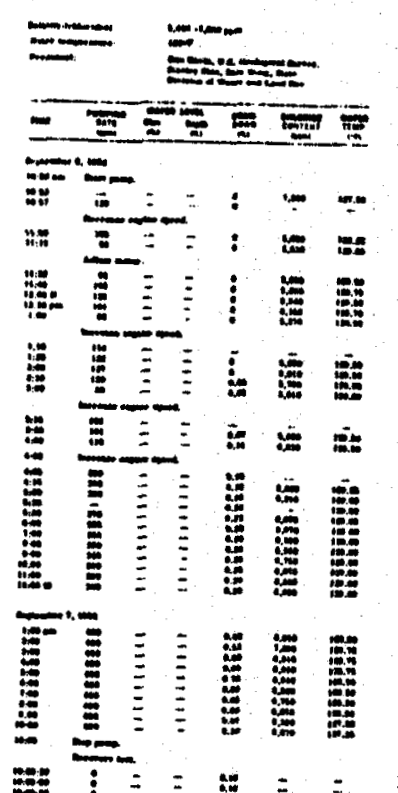

푼
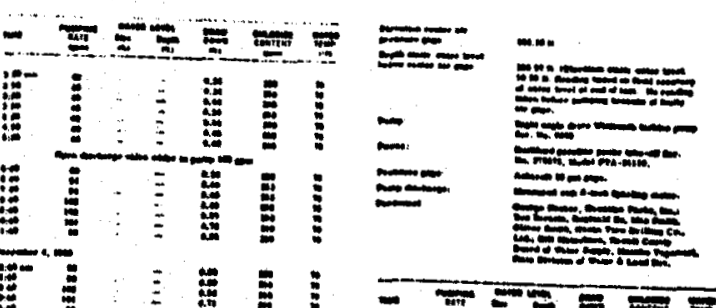

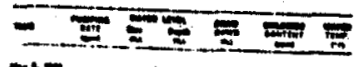

$\min :=-1$

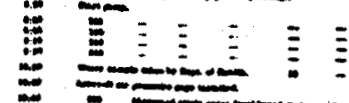

(1)

$=$

(1)

(1)
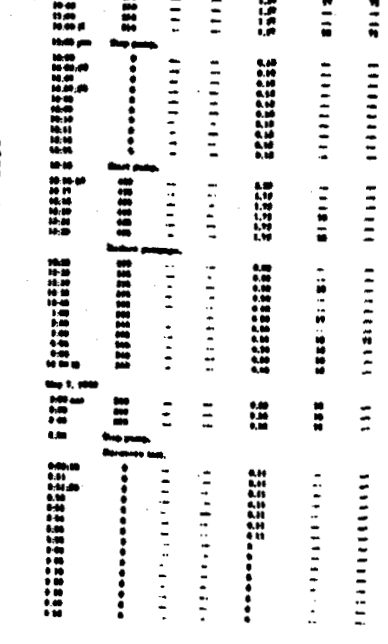

mares nomy

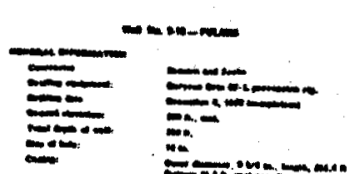

in

$\equiv$

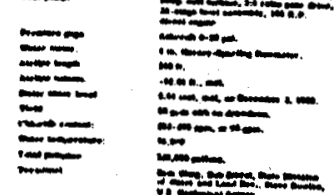

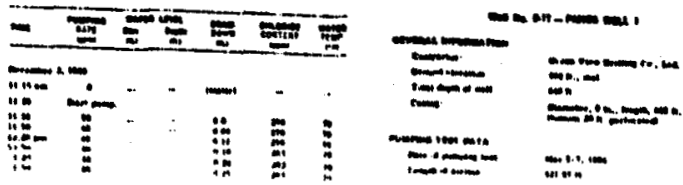

$\|$

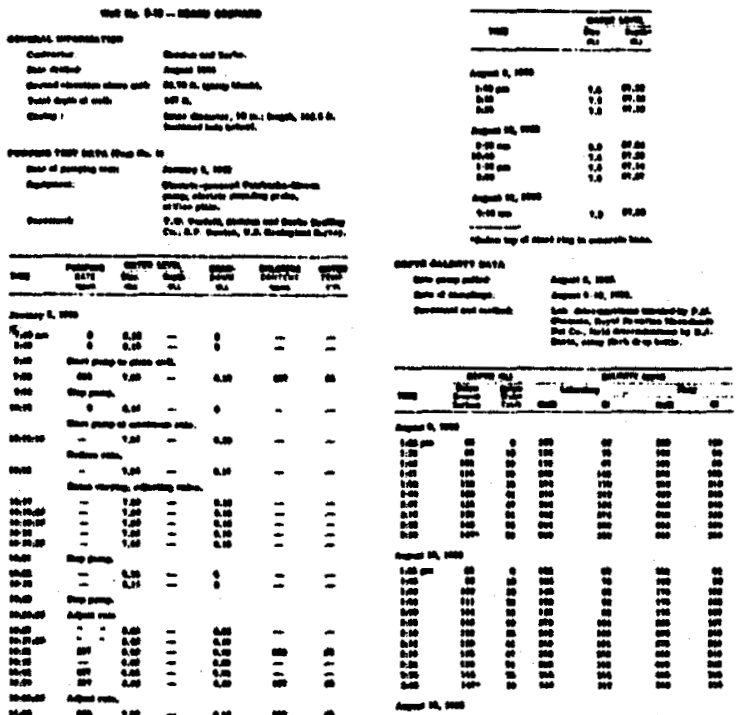

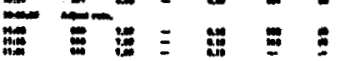

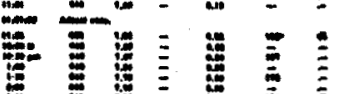

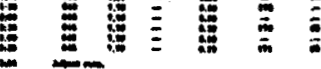

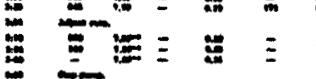

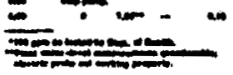

$\equiv$

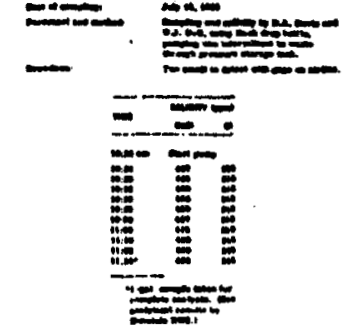

minn

遂要

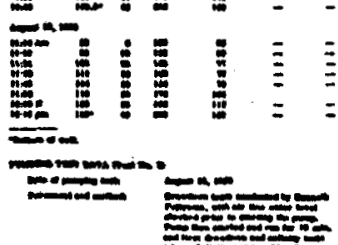

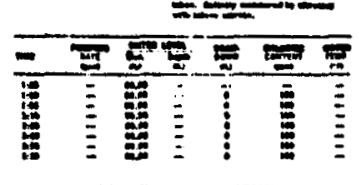

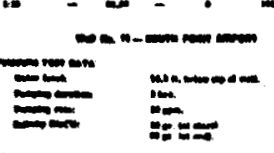

$\equiv$

$\equiv=$

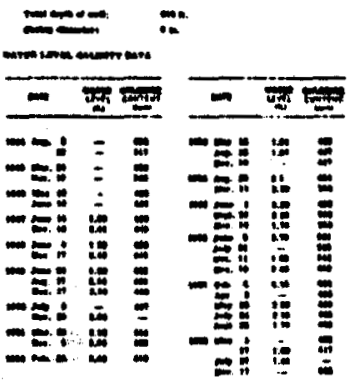

$==-$

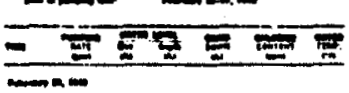

F泣:

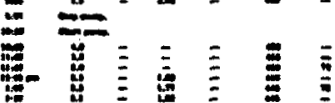

5

$\bar{E}$

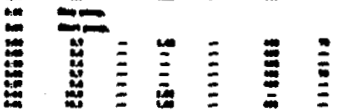

$\underline{E}$

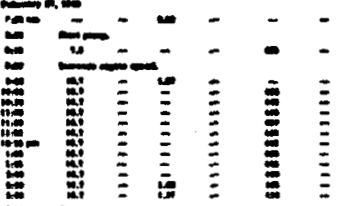

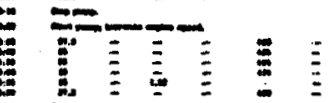

$=$

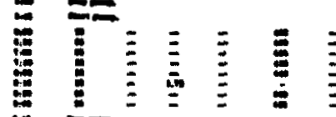

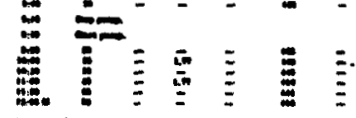

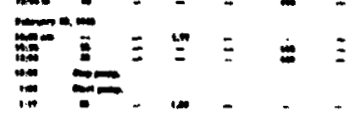

